Original Paper

\title{
Determination of Trace Amounts of Chiral Amino Acids in Complicated Biological Samples Using Two-Dimensional High-Performance Liquid Chromatography with an Innovative "Shape-Fitting" Peak Identification/Quantification Method
}

\author{
Kenji HAMASE*1, Tatsuhiko IKEDA ${ }^{2}$, Chiharu ISHII ${ }^{1}$, Shoto ISHIGO², Kei MASUYAMA², Takeyuki \\ AKITA $^{1}$, Aogu FURUSHO ${ }^{1}$, Miho TAKAHASHI ${ }^{1}$, Tomomi IDE ${ }^{3}$, Masashi MITA ${ }^{2}$ \\ ${ }^{1}$ Graduate School of Pharmaceutical Sciences, Kyushu University, 3-1-1 Maidashi, Higashi-ku, Fukuoka 812-8582, Japan \\ ${ }^{2}$ KAGAMI LAB., Shiseido Global Innovation Center, 1-6-2 Higashi-shimbashi, Minato-ku, Tokyo 105-8310, Japan \\ ${ }^{3}$ Graduate School of Medical Sciences, Kyushu University, 3-1-1 Maidashi, Higashi-ku, Fukuoka 812-8582, Japan
}

\begin{abstract}
A new peak identification/quantification method, i.e., "shape-fitting" method, has been devised and applied to the determination of chiral amino acids in human plasma and urine. The shape fitting method enables the determination of target analytes using a part of the peak by mathematically predicting the whole peak even when the baseline is not clear. Using this method, D-glutamic acid (Glu) and D-proline (Pro) as well as their L-enantiomers in the plasma and urine were determined. The calibration lines of 4 target enantiomers were linear with correlation coefficients higher than 0.9998 ; the RSD values of the intra-day precision and inter-day precision were less than $6.70 \%$. In the human plasma, trace amount of D-Pro $(0.47$ $\mathrm{nmol} / \mathrm{mL}$ ) was observed, and in the urine, $2.97 \mathrm{nmol} / \mathrm{mL}$ of D-Glu and $0.08 \mathrm{nmol} / \mathrm{mL}$ of D-Pro were successfully determined.
\end{abstract}

Keywords: Chiral amino acid; Enantiomer separation; Shape fitting; 2D-HPLC

\section{Introduction}

Chiral amino acids are now increasingly recognized as new biologically-active substances and/or biomarkers [1-4]. Although it is long believed that only L-amino acids are present in the higher animals, several D-amino acids in their free forms were found in mammals including humans. D-Serine (Ser) was found in the frontal brain areas and clarified to modulate neurotransmission via the $\mathrm{N}$-methyl-D-aspartate (NMDA) subtype of the glutamate (Glu) receptors [5,6]. D-Ser also plays an essential role in acquiring cerebellar memory [7]. D-Aspartic acid (Asp) was found in various endocrine tissues and reported to regulate hormonal synthesis/secretion [8]. Correlation between the amounts of the intrinsic D-amino acids and various diseases, such as neuronal diseases and renal disorders, has also been clarified [9-12]. In the spinal cord of mice with amyotrophic lateral sclerosis, the amounts of D-Ser significantly increased [11]. In the plasma of patients with chronic kidney disease, the amounts of D-Ala, D-Asn, D-Pro and D-Ser increased depending on the progression of the disease [12]. The lines of research clearly indicated that the enantioselective determination of chiral amino acids is one of the novel approaches to discover new drug candidates and biomarkers.

For the determination of amino acid enantiomers, a variety of analytical methods using gas chromatography (GC), high-performance liquid chromatography (HPLC) and high-performance capillary electrophoresis (HPCE) has

Received: 31 August 2018

Accepted: 26 September 2018

J-STAGE Advance Published: 12 October 2018

DOI: $10.15583 /$ jpchrom.2018.019 
been reported [13-15]. Concerning GC [13] and HPLC [14], the methods using chiral derivatization reagents or chiral stationary phases are frequently used, and the methods using chiral mobile phase additives are mostly used for HPCE [15]. Among them, multi-dimensional HPLC methods, such as the HPLC-MS (or MS/MS) methods and LC/LC methods, are widely considered to be effective for the sensitive and selective determination of chiral amino acids [2,3]. Especially, two-dimensional (2D) HPLC methods have high peak capacities to separate the compounds and are used in many research studies with various column combinations [16-25]. However, in the complex biological matrices, the determination of trace levels of amino acid enantiomers is difficult due to the co-elution of several known/unknown intrinsic compounds even using the 2D-HPLC methods. After thorough investigations of separation conditions in both stationary phases and mobile phases, interfering compounds are still frequently observed and the target chiral amino acids are only partly separated in some cases. Therefore, a method that enables the determination of these trace compounds without completely separating the target peaks from interfering compounds is eagerly expected.
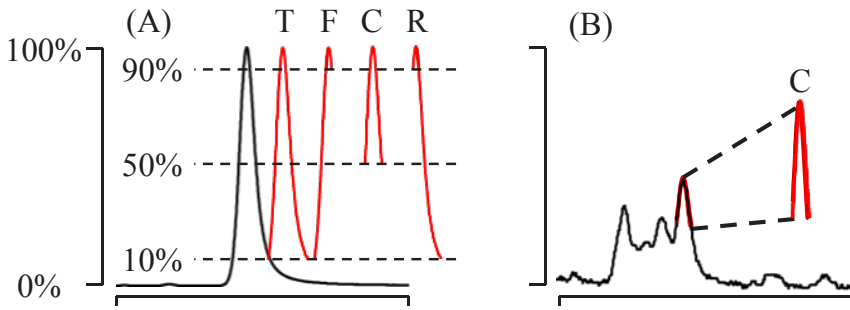

Fig. 1. The outline of the shape fitting method. (A) Four sections including peak top were assigned as total $(\mathrm{T})$, front $(\mathrm{F})$, center (C) and rear (R). (B) The target peak in the complex biological matrices could be identified and quantified using the center part of the peak.

The aim of the present research is to devise a method called the "Shape-Fitting" method that enables the identification/quantification of target compounds when at least a part of the peak is separated from the interfering compounds. The outline of the shape-fitting method is illustrated in Fig. 1. The concept of this method is to identify the target peak by using the shape of the peak because the peak shape of the target compound is identical and only magnified in the direction of the y axis (only the intensity of the detector response is changed). Therefore, the whole target peak is predictable at least when part of the peak (for example, front part $(\mathrm{F})$, center part $(\mathrm{C})$ or rear part $(\mathrm{R})$ ) is observed. As shown in Fig. 1B, the target peak in a complex biological matrix could be identified and quantified just by using the center part of the peak even in the case when the baseline is unclear. In the present paper, the shape-fitting method was validated using the 2D-HPLC analysis of the Glu and Pro enantiomers, and applied to the determination of trace levels of D-Pro in human plasma and also D-Glu and D-Pro in human urine. To the best of our knowledge, this is the first paper to report the shape-fitting method that quantifies the target compounds by using a part of the peak shape. The present concept is practically useful especially for the determination of trace levels of compounds in complex real world samples.

\section{Experimental}

\subsection{Materials}

D-Glu, L-Glu, D-Pro and L-Pro were purchased from FUJIFILM Wako Pure Chemical Corporation (Osaka, Japan). The fluorescence derivatization reagent for the amino group, 4-fluoro-7-nitro-2,1,3-benzoxadiazole (NBD-F), was obtained from Tokyo Chemical Industry (Tokyo, Japan). The LC-MS grade solvents, methanol $(\mathrm{MeOH})$ and acetonitrile $(\mathrm{MeCN})$, were the products of Merck (Darmstadt, Germany). The mobile phase additives, trifluoroacetic acid (TFA) and formic acid (FA), were obtained from FUJIFILM Wako Pure Chemical Corporation. Water was purified by a Milli-Q Integral 3 system (Merck). All other reagents and solvents were the highest grade and used without further purification. Human clinical samples (plasma and urine) were collected from healthy volunteers after informed consent (the clinical experiments were approved by the review board of the Clinical Research Network Fukuoka, No. 14-E05).

\subsection{Sample preparation}

Human plasma was mixed with 20 volumes of $\mathrm{MeOH}$ and centrifuged $(12,000 \mathrm{~g} \times 5 \mathrm{~min})$ to obtain the supernatant The supernatant $(50 \mu \mathrm{L})$ was evaporated to dryness under reduced pressure, and to the residue, $10 \mu \mathrm{L}$ of $400 \mathrm{mM}$ sodium borate buffer $(\mathrm{pH} 8.0)$ and $10 \mu \mathrm{L}$ of $\mathrm{H}_{2} \mathrm{O}$ were added. To the mixture, $5 \mu \mathrm{L}$ of $40 \mathrm{mM}$ NBD-F in MeCN was added, then heated at $60^{\circ} \mathrm{C}$ for $2 \mathrm{~min}$. An aqueous solution of $0.2 \%$ TFA $(75 \mu \mathrm{L})$ was added and the reaction mixture $(10 \mu \mathrm{L})$ was injected into the HPLC systems shown in sections 2.3 and 2.5. The urine sample was diluted 5 times with $\mathrm{H}_{2} \mathrm{O}$ and $10 \mu \mathrm{L}$ of the solution was added to 10 $\mu \mathrm{L}$ of $400 \mathrm{mM}$ sodium borate buffer $(\mathrm{pH} 8.0)$, then derivatized in the same manner as that for the plasma samples. For analysis of the standard amino acids, $10 \mu \mathrm{L}$ of the aqueous standard amino acid solution was added to $10 \mu \mathrm{L}$ of the $400 \mathrm{mM}$ sodium borate buffer ( $\mathrm{pH} \mathrm{8.0)}$ and derivatized as already described.

\subsection{D-HPLC system for the chiral analysis of Glu and Pro}

The 2D-HPLC analysis of the Glu and Pro enantiomers was carried out by an originally-designed HPLC system combining the Nanospace SI-2 HPLC apparatus (Shiseido, 
Tokyo, Japan). The system consisted of a 3202 degasser, 3101 and 3201 pumps, a 3023 auto-sampler, 3004 and 3014 column ovens, a 3012 high-pressure 6-port valve, a 9986 multi-loop valve with the loops of $650 \mu \mathrm{L}$, a $3002 \mathrm{UV}$ detector and a 3013 fluorescence detector. The separation column for the first dimension was a KSAARP column (1.0 $\mathrm{mm}$ i.d. $\mathrm{x} 500 \mathrm{~mm}, 3 \mu \mathrm{m}$, an ODS column designed by the collaboration with Shiseido, $45^{\circ} \mathrm{C}$ ) and the elution of NBD-Glu was carried out by an aqueous solution containing $10 \% \mathrm{MeCN}$ and $0.05 \%$ TFA at the flow rate of $25 \mu \mathrm{L} / \mathrm{min}$. The elution of NBD-Pro was carried out by an aqueous solution containing $20 \% \mathrm{MeCN}$ and $0.05 \%$ TFA. Elution of the NBD-amino acids was monitored by the absorbance at $470 \mathrm{~nm}$, and the target fractions were introduced into the second dimension.

The analytical column for the second dimension was a KSAACSP-001S $(1.5 \mathrm{~mm}$ x $250 \mathrm{~mm}, 5 \mu \mathrm{m}$, a Pirkle-type enantioselective column designed by collaboration with Shiseido, having (3,5-dinitrophenylaminocarbonyl)-L-Leu as a chiral selector, $25^{\circ} \mathrm{C}$ ). The elution of NBD-Glu was performed using a mixture of $\mathrm{MeCN}-\mathrm{MeOH}(80 / 20, \mathrm{v} / \mathrm{v})$ containing $0.3 \%$ FA, and the elution of NBD-Pro was performed by a mixture of $\mathrm{MeCN}-\mathrm{MeOH}(20 / 80, \mathrm{v} / \mathrm{v})$ containing $0.2 \% \mathrm{FA}$. The flow rate of the mobile phase was $150 \mu \mathrm{L} / \mathrm{min}$, and the detection of the NBD-amino acid enantiomers was carried out by the fluorescence at $530 \mathrm{~nm}$ with excitation at $470 \mathrm{~nm}$.

\subsection{Shape-fitting method for the identification and quantification of the target peaks}

Target peaks are calibrated by standard peak shape scaling. This method is different from conventional baseline-corrected area/height calculation and also different from prediction of the known peak combinations. All fluorescence detection data $(5 \mathrm{~Hz})$ were output as ASCII format files by EZChrom. The detector signal was obtained every $0.2 \mathrm{sec}$. To identify the peaks for the standard amino acids, the points having intensities higher than 10 times those of baseline noise (difference between maximum and minimum intensities of 150 continuous data points in the stable area close to the target peak) were used. To the obtained data points of the peaks, four sections including the peak top were assigned as "Total" (10 - 100 10\%, Fig. 1, (A)-T), "Front" (10 - $100-90 \%$, Fig. 1, (A)-F), "Center" (50 - $100-50 \%$, Fig. 1, (A)-C) and "Rear" (90 $100-10 \%$, Fig. 1, (A)-R). In order to make calibration lines, the standard amino acids with various concentrations were analyzed, and the obtained data points were multiplied by a "constant" so that the peak shape was the same as that of the reference peak analyzing $1 \mathrm{pmol}$ (per injection) of the target amino acid enantiomers. Using the obtained "constant", the calibration lines were made against the amounts of the injected target amino acids. In principle, the calibration lines could be made using all of the 4 sections ( $\mathrm{T}, \mathrm{F}, \mathrm{C}$ and $\mathrm{R}$ ), and the 4 lines have the same meaning.

The "shape-fitting" of the target analytes in the real-world samples was performed by the following steps. The sample was analyzed using the 2D-HPLC system shown in section 2.3., and the peak sections (T, F, C and R, shown in Fig. 1) of the target analyte were assigned (where the interference was not severe). To the obtained peak sections, the peak shape obtained by the standard amino acid enantiomer was superimposed, and the "magnification constant" best fitted to the target peak in the real-world sample was identified. Consequently, the concentration of the target analytes were calculated by using the identified magnification constant and the calibration line made by the standard amino acid enantiomers.

\subsection{D HPLC-MS/MS system for the chiral analysis of Glu and Pro}

The 2D HPLC-MS/MS analysis of the NBD-Glu and NBD-Pro enantiomers was performed using the same HPLC system described in Section 2.3., combining a tandem mass spectrometer (Triple Quad ${ }^{\mathrm{TM}}$ 5500, Sciex, Framingham, MA, USA). The separation columns and mobile phases for both dimensions were also the same as described in Section 2.3. The precursor and product ions for NBD-Glu were 311/247 and those for NBD-Pro were 279/217. The conditions for the MS/MS were as follows: ion spray voltage, $5500 \mathrm{~V}$; ion source gas 1,50 psi (345 $\mathrm{kPa})$; ion source gas 2, $50 \mathrm{psi}(345 \mathrm{kPa})$; curtain gas, $20 \mathrm{psi}$ $(138 \mathrm{kPa})$; collision gas, $8 \mathrm{psi}(55 \mathrm{kPa})$; ion source temperature, $650^{\circ} \mathrm{C}$; and entrance potential, $10 \mathrm{~V}$. The declustering potentials were $131 \mathrm{~V}$ and $95 \mathrm{~V}$, the collision energies were $21 \mathrm{eV}$ and $27 \mathrm{eV}$, and the collision cell exit

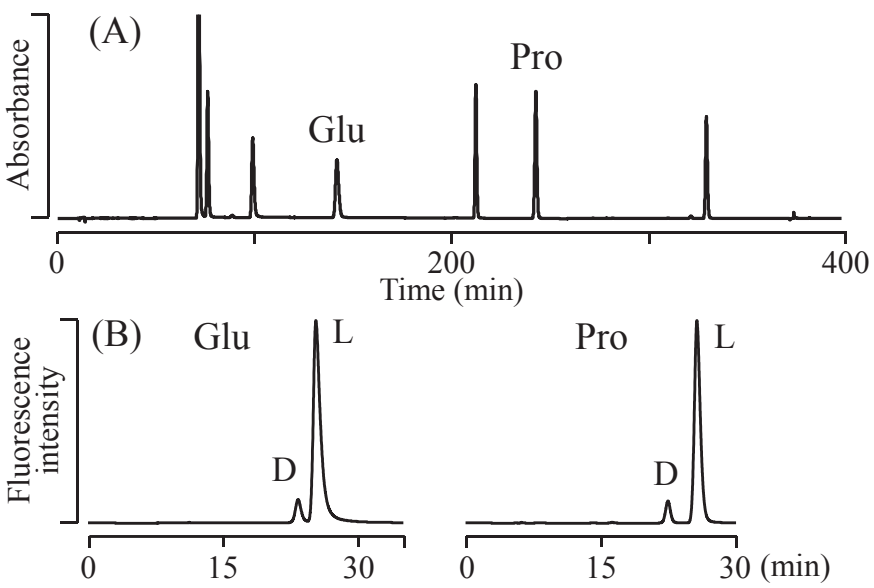

Fig. 2. (A) Reversed-phase separation of the standard NBD-Glu and NBD-Pro by the KSAARP column. (B) Enantiomer separations of the mixtures of the D-forms and L-forms in the molar ratio of $1: 10$ by the KSAACSP-001S column. The detailed separation conditions are described in the text. 
potentials were $20 \mathrm{~V}$ and $16 \mathrm{~V}$ for Glu and Pro, respectively. For the data processing, the Analyst system (Sciex) was used.

\section{Results and discussion}

3.1. 2D-HPLC separation of NBD-Glu and NBD-Pro enantiomers and validation results using shape-fitting peak identification/quantification method

As an example to show the applicability of the shape-fitting peak identification/purification method, the enantiomers of Glu and Pro were analyzed using a 2D-HPLC system combining ODS and enantioselective columns. As the target chiral amino acids, Glu and Pro were selected because the amounts of these two D-amino acids are trace in human plasma and urine, and it is sometimes difficult to separate these chiral amino acids completely from other intrinsic substances [26]. Figure $2 \mathrm{~A}$ shows the reversed-phase HPLC separation of the standard NBD-Glu and NBD-Pro in the presence of several proteinogenic amino acids (in this case, Ala, Asp, Leu and Ser; major proteinogenic amino acids that the D-forms are frequently observed in biological samples). In the reversed-phase HPLC (first dimension), NBD derivatives of Glu and Pro were nicely separated from the other amino acids within about $240 \mathrm{~min}$. The target fractions were collected to the multi-loop device, then on-line automatically introduced into the second dimension. An original Pirkle-type chiral stationary phase, KSAACSP-001S $(1.5 \mathrm{~mm} \times 250 \mathrm{~mm})$, was placed in the second dimension, and the enantiomers of NBD-Glu and NBD-Pro were baseline separated within $30 \mathrm{~min}$ (Fig. 2B).

The validation results of the present shape-fitting method were evaluated by the calibration lines, intra-day precision and inter-day precision using standard amino acid enantiomers. As shown in Table 1, all of the calibration lines for the Glu and Pro enantiomers constructed using 4 sections of the peak shape (T, F, C and R) were linear with correlation coefficients higher than 0.9998 . The intra-day precision and inter-day precision were determined by analyzing the D-Glu (0.05 and $1.0 \mathrm{pmol} /$ injection), D-Pro (0.05 and $1.0 \mathrm{pmol} / \mathrm{injection})$, L-Glu $(0.5$ and 10 $\mathrm{pmol} /$ injection) and L-Pro (0.5 and $10 \mathrm{pmol} /$ injection). The obtained RSD values were lower than 6.70 , indicating that the present shape-fitting method has sufficient linearity and precision.

In the present study, a new peak identification/ quantification concept using the shape-fitting method for the determination of chiral amino acids has been reported. For the quantification of trace levels of the D-forms in real-world samples, the separation is not sufficient and the baseline is not clear in many cases. Therefore, a method which enables the peak identification/quantification using only a part of the peak is practically highly expected.
Table 1. Calibration lines of standard amino acids and precision of the method.

\begin{tabular}{|c|c|c|c|c|c|c|c|c|}
\hline \multirow{3}{*}{$\begin{array}{c}\text { Amino } \\
\text { acid }\end{array}$} & \multicolumn{4}{|c|}{ Calibration line } & \multicolumn{4}{|c|}{ Precision (RSD, \%) } \\
\hline & \multirow{2}{*}{$\begin{array}{l}\text { Calibration } \\
\text { range }\end{array}$} & \multirow{2}{*}{$\begin{array}{c}\text { Fitting } \\
\text { type }\end{array}$} & \multirow{2}{*}{ Equation } & \multirow{2}{*}{$\begin{array}{l}\text { Correlation } \\
\text { coefficient }\end{array}$} & \multicolumn{2}{|c|}{ Intra-day } & \multicolumn{2}{|c|}{ Inter-day } \\
\hline & & & & & Low & High & Low & High \\
\hline \multirow{4}{*}{ D-Glu } & \multirow{4}{*}{$0.005-1$} & $\mathrm{~T}$ & $y=1.0034 x+0.002$ & 0.9998 & 1.60 & 2.62 & 4.86 & 3.59 \\
\hline & & $\mathrm{F}$ & $y=1.0084 x+0.002$ & 0.9998 & 1.95 & 3.34 & 4.11 & 3.64 \\
\hline & & $\mathrm{C}$ & $y=1.0064 x+0.006$ & 0.9998 & 2.25 & 3.41 & 5.21 & 4.68 \\
\hline & & $\mathrm{R}$ & $y=1.0056 x+0.000$ & 0.9998 & 1.70 & 2.68 & 3.53 & 3.55 \\
\hline \multirow{4}{*}{ L-Glu } & \multirow{4}{*}{$0.05-1$} & $\mathrm{~T}$ & $y=0.9941 x+0.007$ & 1.0000 & 0.66 & 2.57 & 3.59 & 4.11 \\
\hline & & $\mathrm{F}$ & $y=1.0004 x+0.008$ & 1.0000 & 1.51 & 1.82 & 2.71 & 3.24 \\
\hline & & $\mathrm{C}$ & $y=0.9898 x+0.003$ & 1.0000 & 0.99 & 2.82 & 5.71 & 3.98 \\
\hline & & $\mathrm{R}$ & $y=0.9942 x+0.007$ & 1.0000 & 1.00 & 2.56 & 3.48 & 3.45 \\
\hline \multirow{4}{*}{ D-Pro } & \multirow{4}{*}{$0.005-1$} & $\mathrm{~T}$ & $y=1.0042 x+0.002$ & 0.9999 & 2.65 & 4.65 & 3.97 & 4.53 \\
\hline & & $\mathrm{F}$ & $y=1.0034 x+0.002$ & 0.9999 & 1.87 & 4.65 & 4.76 & 4.49 \\
\hline & & $\mathrm{C}$ & $y=1.0119 x+0.007$ & 0.9999 & 3.06 & 4.79 & 3.76 & 4.61 \\
\hline & & $\mathrm{R}$ & $y=1.0061 x+0.003$ & 0.9999 & 2.02 & 3.79 & 4.41 & 4.01 \\
\hline \multirow{4}{*}{ L-Pro } & \multirow{4}{*}{$0.05-10$} & $\mathrm{~T}$ & $y=0.9972 x+0.008$ & 0.9999 & 1.78 & 4.46 & 4.95 & 5.05 \\
\hline & & $\mathrm{F}$ & $y=0.9930 x+0.009$ & 0.9999 & 2.27 & 4.31 & 3.71 & 4.87 \\
\hline & & $\mathrm{C}$ & $y=0.9678 x+0.059$ & 0.9999 & 2.48 & 6.08 & 3.64 & 6.70 \\
\hline & & $\mathrm{R}$ & $y=1.0121 x+0.016$ & 0.9999 & 2.60 & 4.68 & 4.53 & 4.38 \\
\hline
\end{tabular}

Equations were made where $\mathrm{x}$ is the amount of the target amino acid (pmol/injection), and $\mathrm{y}$ is the magnification constant of the peak. Injection amounts of the D-amino acids are $0.005,0.01,0.05,0.1$, 0.5 and 1 pmol. Those of L-Glu are $0.05,0.1,0.5$ and 1 pmol. Those of L-Pro are $0.05,0.1,0.5,1,5$ and 10 pmol. For the precision $(\mathrm{n}=4)$ of the standard amino acids, $50 \mathrm{fmol}$ and $1 \mathrm{pmol}$ of the D-amino acids and $500 \mathrm{fmol}$ and $10 \mathrm{pmol}$ of the L-amino acids were injected.

By the shape-fitting method proposed in the present study, the calibration lines constructed by the 4 sections of the peaks (T, F, C and R sections) were linear, and the RSD values of the intra-day precision and inter-day precision were also sufficient. These results indicated that the present shape-fitting method is practically useful, and the applicability to human clinical samples was evaluated in Section 3.2.

\subsection{Determination of trace levels of D-Glu and D-Pro in the human plasma and urine using the shape-fitting method}

As an application of the shape-fitting method, the enantiomers of Glu and Pro in the human plasma and urine were determined. Figure 3 shows the chromatograms obtained for the human plasma. In the reversed-phase separation, the fractions of Glu and Pro were trapped in the multi-loop valve, and were on-line injected into the enantioselective column in the same manner as for the standard amino acids. In the second dimension, a trace peak of D-Pro and large peaks of L-Glu and L-Pro were observed in the human plasma. In this case, the amounts of D-Pro and also L-forms of Glu and Pro were determined by the shape-fitting method using the frontal part of the peaks. The obtained values were $0.47 \mathrm{nmol} / \mathrm{mL}$ plasma for D-Pro and 13.92 and $64.62 \mathrm{nmol} / \mathrm{mL}$ plasma for the L-Glu and L-Pro, respectively. As a more complicated biological matrix, human urine was selected and analyzed by the same 2D-HPLC system. The obtained chromatograms are shown in Fig. 4. In the reversed-phase separation (Fig. 4A), many peaks were observed, and the 

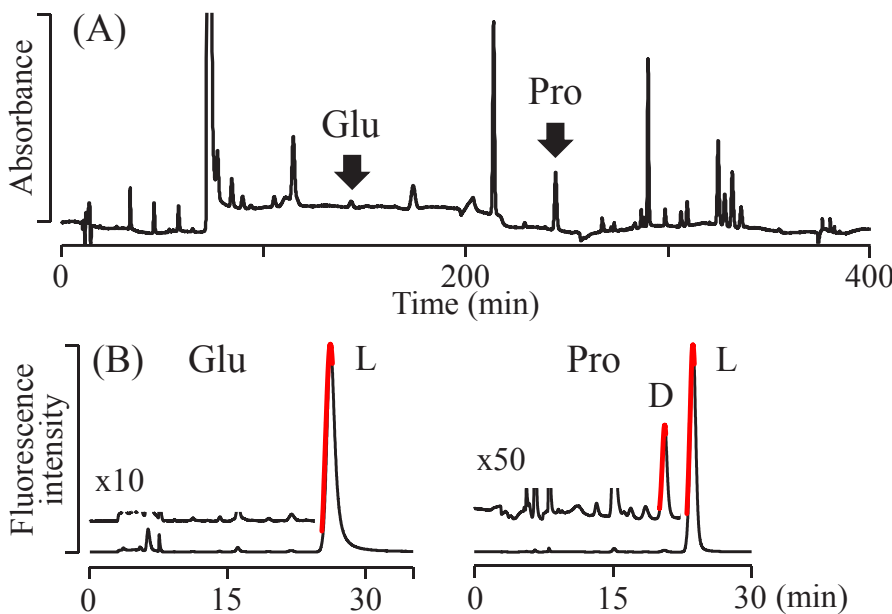

Fig. 3. (A) Reversed-phase separation of NBD-Glu and NBD-Pro in human plasma. (B) Enantioselective determination of NBD-Glu and NBD-Pro by a fluorescence detector. The red lines indicate the superimposed fitting peaks.

fractions of NBD-Glu and Pro were collected using the retention time. In the second dimension, although the number of peaks was less than that in the first dimension, several unknown peaks were still co-eluted with the target chiral amino acids. In this case, the amounts of D-Glu and D-Pro were determined by the shape-fitting method using the center part of the peaks, and those of the L-forms were determined using the rear part. The obtained values were $2.97 \mathrm{nmol} / \mathrm{mL}$ urine for D-Glu, $12.81 \mathrm{nmol} / \mathrm{mL}$ for L-Glu, $0.08 \mathrm{nmol} / \mathrm{mL}$ for D-Pro and $5.57 \mathrm{nmol} / \mathrm{mL}$ for L-Pro.

To show the validation results of the shape-fitting method for the analysis of real biological samples, the intra-day precision and accuracy were checked using human plasma and urine. As shown in Table 2, the RSD values of the intra-day precision of the intrinsic chiral amino acids in the human plasma were lower than $5.76 \%$, and those in the human urine were lower than $7.84 \%$. Accuracy values were $96.2-106.9 \%$. These results indicated that the present peak fitting method has practically sufficient precision and accuracy. In order to check the amounts of the chiral amino acids determined by the 2D-HPLC system using the shape-fitting data-processing approach, the same samples were determined by the 2D HPLC-MS/MS system combining the same 2D-HPLC system and triple quadrupole tandem mass spectrometry. The results are shown in Fig. 5. By the 2D HPLC-MS/MS system, no interfering peaks were present, and only the target chiral amino acid peaks were observed. The obtained amounts of these chiral amino acids were $0.43 \mathrm{nmol} / \mathrm{mL}$ plasma for D-Pro, $12.88 \mathrm{nmol} / \mathrm{mL}$ for L-Glu and $56.84 \mathrm{nmol} / \mathrm{mL}$ for L-Pro. In the urine, the amounts were $2.58 \mathrm{nmol} / \mathrm{mL}$ for D-Glu, $15.32 \mathrm{nmol} / \mathrm{mL}$ for L-Glu, $0.04 \mathrm{nmol} / \mathrm{mL}$ for D-Pro and $6.63 \mathrm{nmol} / \mathrm{mL}$ for L-Pro. These values were almost consistent with those obtained by the shape-fitting method.
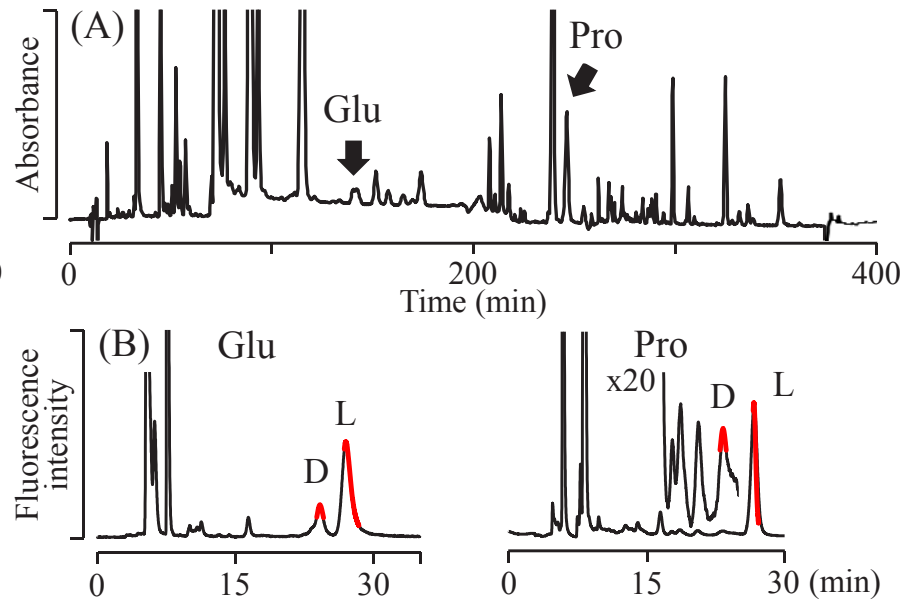

Fig. 4. (A) Reversed-phase separation of NBD-Glu and NBD-Pro in human urine. (B) Enantioselective determination of NBD-Glu and NBD-Pro by a fluorescence detector. The red lines indicate the superimposed fitting peaks.

Table 2. Precision and accuracy of the method using the human plasma and urine.

\begin{tabular}{ccccccccc}
\hline & \multicolumn{3}{c}{ Plasma } & & \multicolumn{3}{c}{ Urine } \\
\cline { 2 - 3 } Amino acid & $\begin{array}{c}\text { Fitting } \\
\text { type }\end{array}$ & $\begin{array}{c}\text { Precision } \\
(\mathrm{RSD}, \%)\end{array}$ & $\begin{array}{c}\text { Accuracy } \\
(\%)\end{array}$ & & $\begin{array}{c}\text { Fitting } \\
\text { type }\end{array}$ & $\begin{array}{c}\text { Precision } \\
(\mathrm{RSD}, \%)\end{array}$ & $\begin{array}{c}\text { Accuracy } \\
(\%)\end{array}$ \\
\hline D-Glu & $\mathrm{F}$ & - & 106.9 & & $\mathrm{C}$ & 7.84 & 105.4 \\
L-Glu & $\mathrm{F}$ & 5.28 & 106.9 & & $\mathrm{R}$ & 5.04 & 103.8 \\
\hline D-Pro & $\mathrm{F}$ & 5.01 & 102.9 & & $\mathrm{C}$ & 3.63 & 96.2 \\
L-Pro & $\mathrm{F}$ & 5.76 & 100.6 & & $\mathrm{R}$ & 7.01 & 98.5 \\
\hline
\end{tabular}

For the precision using the human plasma, the intrinsic amino acids were determined. For the accuracy, 8 pmol of the D-amino acids and $80 \mathrm{pmol}$ of the L-amino acids were added to the plasma. For the precision using the human urine, intrinsic amino acids were determined. For the accuracy, 50 pmol of racemic amino acids were added to the urine.
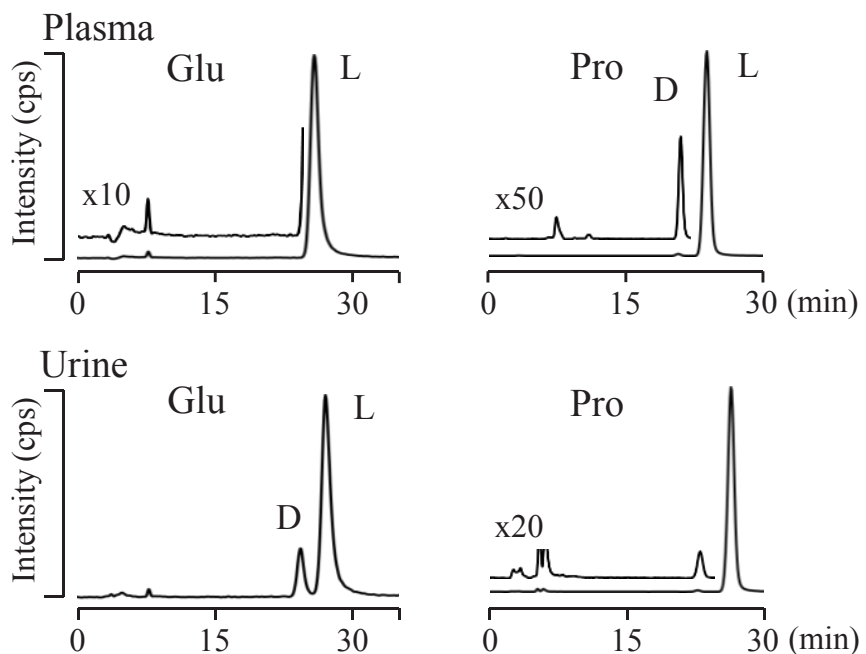

Fig. 5. Enantioselective determination of NBD-Glu and NBD-Pro in human plasma and urine by the 2D-HPLC-MS/MS system. 
Several papers have been published reporting the amounts of D-amino acids in the plasma and urine of mammals [12,17,22,26-29]. In the mouse, the tissue distribution of D-Glu [22] and D-Pro [17], and their concentrations in the physiological fluids have been reported. In the mouse, the plasma concentration of D-Glu was trace, and that of $\mathrm{D}$-Pro was $0.7 \mathrm{nmol} / \mathrm{mL}$. In the urine, D-Glu was $3.7 \mathrm{nmol} / \mathrm{mL}$ and D-Pro was 23.2 $\mathrm{nmol} / \mathrm{mL}$. In the human plasma, the amount of D-Pro was also trace, and that of D-Pro was $0.24-3.5 \mathrm{nmol} / \mathrm{mL}$ [12,26-29].

In the human urine, the amount of D-Glu was $3.9 \mathrm{nmol} / \mathrm{mL}$ [26] and that of D-Pro was $0.004-4.0 \mathrm{nmol} / \mathrm{mL}$ [26-29]. Although a strain dependent difference was observed in the concentration of D-Pro in the urine, the other values are almost consistent. These results indicated that the trace levels of the D-amino acids in complicated biological samples could be determined by the present shape-fitting method, and further applications are in progress.

\section{Conclusion}

In the present study, a new concept, i.e., the shape-fitting method, for the peak identification/quantification was designed and evaluated. The method was successfully applied to the determination of trace amounts of D-amino acids in complicated biological samples. Because the chiral amino acid analysis was frequently disturbed by the co-elution with various known/unknown intrinsic substances, the present approach is practically useful in a variety of clinical applications.

\section{References}

[1] Fujii, N.; Kaji, Y.; Fujii, N. J. Chromatogr. B 2011, 879, 3141-3147.

[2] Miyoshi, Y.; Koga, R.; Oyama, T.; Han, H.; Ueno, K.; Masuyama, K.; Itoh, Y.; Hamase, K. J. Pharm. Biomed. Anal. 2012, 69, 42-49.

[3] Miyoshi, Y.; Oyama, T.; Itoh, Y.; Hamase, K. Chromatography 2014, 35, 49-57.

[4] Ishii, C.; Miyamoto, T.; Ishigo, S.; Miyoshi, Y.; Mita, M.; Homma, H.; Ueda, T.; Hamase, K. Chromatography 2017, 38, 65-72.

[5] Henneberger, C.; Papouin, T.; Oliet, S. H. R.; Rusakov, D. A. Nature 2010, 463, 232-236.

[6] Nishikawa, T. J. Chromatogr. B 2011, 879, 3169-3183.

[7] Kakegawa, W.; Miyoshi, Y.; Hamase, K.; Matsuda, S.; Matsuda, K.; Kohda, K.; Emi, K.; Motohashi, J.; Konno, R.; Zaitsu, K.; Yuzaki, M. Nat. Neurosci. 2011, 14, 603-611.

[8] Katane, M.; Homma, H. J. Chromatogr. B 2011, 879, 3108-3121.

[9] Fisher, G.; Lorenzo, N.; Abe, H.; Fujita, E.; Frey, W.
H.; Emory, C.; Di Fiore, M. M.; D'Aniello, A. Amino Acids 1998, 15, 263-269.

[10] Bendikov, I.; Nadri, C.; Amar, S.; Panizzutti, R.; De Miranda, J.; Wolosker, H.; Agam, G. Schizophr. Res. 2007, 90, 41-51.

[11] Sasabe, J.; Miyoshi, Y.; Suzuki, M.; Mita, M.; Konno, R.; Matsuoka, M.; Hamase, K.; Aiso, S. Proc. Natl. Acad. Sci. USA 2012, 109, 627-632.

[12] Kimura, T.; Hamase, K.; Miyoshi, Y.; Yamamoto, R.; Yasuda, K.; Mita, M.; Rakugi, H.; Hayashi, T.; Isaka, Y. Sci. Rep. 2016, 6, 26137.

[13] Schurig, V. J. Chromatogr. B 2011, 879, 3122-3140.

[14] Hamase, K.; Morikawa, A.; Zaitsu, K. J. Chromatogr. B 2002, 781, 73-91.

[15] Kitagawa, F.; Otsuka, K. J. Chromatogr. B 2011, 879, 3078-3095.

[16] Morikawa, A.; Hamase, K.; Zaitsu, K. Anal. Biochem. 2003, 312, 66-72.

[17] Hamase, K.; Takagi, S.; Morikawa, A.; Konno, R.; Niwa, A.; Zaitsu, K. Anal. Bioanal. Chem. 2006, 386, 705-711.

[18] Hamase, K.; Morikawa, A.; Ohgusu, T.; Lindner, W.; Zaitsu, K. J. Chromatogr. A 2007, 1143, 105-111.

[19] Miyoshi, Y.; Hamase, K.; Tojo, Y.; Mita, M.; Konno, R.; Zaitsu, K. J. Chromatogr. B 2009, 877, 2506-2512.

[20] Hamase, K.; Miyoshi, Y.; Ueno, K.; Han, H.; Hirano, J.; Morikawa, A.; Mita, M.; Kaneko, T.; Lindner, W.; Zaitsu, K. J. Chromatogr. A 2010, 1217, 1056-1062.

[21] Miyoshi, Y.; Hamase, K.; Okamura, T.; Konno, R.; Kasai, N.; Tojo, Y.; Zaitsu, K. J. Chromatogr. B 2011, 879, 3184-3189.

[22] Han, H.; Miyoshi, Y.; Ueno, K.; Okamura, C.; Tojo, Y.; Mita, M.; Lindner, W.; Zaitsu, K.; Hamase, K. J. Chromatogr. B 2011, 879, 3196-3202.

[23] Koga, R.; Miyoshi, Y.; Sato, Y.; Mita, M.; Konno, R.; Lindner, W.; Hamase, K. Chromatography 2016, 37, $15-22$.

[24] Koga, R.; Miyoshi, Y.; Sato, Y.; Mita, M.; Konno, R.; Lindner, W.; Hamase, K. J. Chromatogr. A 2016, 1467, 312-317.

[25] Morikawa, A.; Fukuoka, H.; Uezono, K.; Mita, M.; Koyanagi, S.; Ohdo, S.; Zaitsu, K.; Hamase, K. Chromatography 2017, 38, 53-58.

[26] Ishii, C. Akita, T., Mita, M., Ide, T., Hamase, K. J. Chromatogr. A, 2018, 1570, 91-98.

[27] Visser, W. F., Verhoeven-Duif, N. M., Ophoff, R., Bakker, S., Klomp, L. W., Berger, R., de Koning, T. J. J. Chromatogr. A 2011, 1218, 7130-7136.

[28] Armstrong, D. W., Gasper, M., Lee, S. H., Zukowski, J., Ercal, N. Chirality 1993, 5, 375-378.

[29] Brückner, H., Schieber, A. Biomed. Chromatogr. 2001, 15, 166-172. 